\title{
EFEITO DA TEMPERATURA E TAXA DE CISALHAMENTO NAS PROPRIEDADES DE ESCOAMENTO DA POLPA DE CUPUAÇU

\author{
(T. grandiflorum Schum) INTEGRAL ${ }^{1}$
}

\author{
GILVANETE MARIA FERREIRA², MARIA JOSÉ DE OLIVEIRACAVALCANTI GUIMARÃES ${ }^{3}$, \\ MARIA CRISTINAANTUN MAIA ${ }^{4}$
}

RESUMO-O comportamento reológico da polpa de cupuaçu integral foi determinado na faixa de temperatura de 10 a $60^{\circ} \mathrm{C}$. Os efeitos da temperatura e da taxa de deformação foram avaliados por meio de testes em cisalhamento estacionário. As análises reológicas foram conduzidas num reômetro Thermo Haake RheoStress 1. Os reogramas foram descritos pelos modelos reológicos de Ostwald-deWaelle e o de Herschel-Bulkley. As curvas de escoamento mais bem ajustadas pelo modelo de Ostwald-de-Waelle. O produto apresentou comportamento pseudoplástico, e o índice de comportamento de fluxo (n) decresceu com o aumento da temperatura. O efeito da temperatura sobre a viscosidade aparente foi descrita por uma equação tipo Arrhenius e discutida em termos de energia de ativação. Essa energia aumentou com o aumento da taxa de deformação, sendo obtidos valores na faixa de 1 a $2 \mathrm{kcal} / \mathrm{gmol}$.

Termos para indexação: cupuaçu, reologia, cisalhamento estacionário, Arrhenius.

\section{EFFECT OF TEMPERATURE AND SHEAR RATE IN THE PROPERTIES OF WHOLE FLOW CUPUASSU PULP (Theobroma grandiflorum)}

ABSTRACT-The rheological behavior of the entire cupuassu pulp was determined in the temperature range $10-60^{\circ} \mathrm{C}$. The effects of temperature and deformation rate were evaluated by means of shear flow tests. Rheometry analyses were conducted with a Thermo Haake RheoStress 1 system. Rheograms were described by models by Ostwald-of-Waelle and Herschel-Bulkley. The Ostwald-ofWaelle model successfully represented pulp flow behavior. The product presented shear-thinning behavior and the flow behavior index (n) decreased with the increase of temperature. The effect of temperature on the apparent viscosity in cupuassu pulp was described by an Arrhenius equation and discussed in terms of activation energy. The increase in shear rate caused an energy increase, and values were obtained in the range of a line from 1 to $2 \mathrm{kcal} / \mathrm{gmol}$.

Index ierms: cupuassu, rheology, shear flow, Arrhenius.

\section{INTRODUÇÃO}

O cupuaçu (T. grandiflorum Schum) é uma das frutas amazônicas mais importantes, sendo o Estado do Pará o principal produtor, seguido pelo Amazonas, Rondônia, Acre, norte do Maranhão e Tocantins. Esse fruto também pode ser encontrado em algumas cidades dos Estados de São Paulo, Bahia e Rio de Janeiro e em outros países, como Colômbia, Venezuela, Equador, Costa Rica, Guiana, São Tomé, Trinidad e Gana (Venturieri, 1993; Lopes, 2000). A polpa é consumida na forma de sucos, néctar, geléias, doces, cremes, tortas, sorvetes, balas, bombons, licores, polpa seca, polpa granulada, entre outras formas.

Os plantios de cupuaçu têm crescido em muitas áreas da Amazônia Brasileira devido ao aumento da demanda pela polpa, que vem sendo exportada, principalmente na forma congelada, para estados do Sudeste do Brasil e para países europeus (Bastos et al., 2002).

O rendimento dos frutos varia de acordo com o tamanho, a procedência, o período de safra e o método de extração. Os frutos pesam em média $1,275 \mathrm{~kg}$ e têm cerca de $43 \%$ de casca,
$38,5 \%$ de polpa, $17,19 \%$ de semente e $2,85 \%$ de placenta. A polpa comestível é de coloração amarela ou esbranquiçada, de sabor ácido e aroma forte, e é muito apreciada organolepticamente (Venturieri, 1993). Do ponto de vista nutritivo, a cada $100 \mathrm{~g}$ de polpa de cupuaçu são encontrados, em média, 14,70 g de glicídios, 1,70 g de proteínas, 1,60 g de lipídeos $1.800 \mathrm{mcg}$ de vitamina B1, $215 \mathrm{mcg}$ de vitamina B2, 3,200 $\mathrm{mg}$ de niacina, 2,60 $\mathrm{mg}$ de ferro, $23 \mathrm{mg}$ de cálcio, $26 \mathrm{mg}$ de fósforo e $26,5 \mathrm{mg}$ de vitamina C (Franco, 1999).

O cupuaçu tem sua produção concentrada no Estado do Pará, tendo grande aumento na área plantada (2.473 a 12.127 ha) e, conseqüentemente, um correspondente aumento na produção (9.737 a 38.488 toneladas), entre 1997 e 2005 ( Sagri, 2006).

Para a indústria de alimentos, o estudo reológico é de grande utilidade em projetos de engenharia de processos, como o dimensionamento de trocadores de calor, sistemas de bombeamento, filtração e concentração (Steffe, 1996), e avaliação de adequação tecnológica (Silva, 2000).

Produtos de frutas e vegetais são considerados como fluidos de comportamento não-newtoniano, seguindo o modelo de Ostwald-de-Waelle ou Lei de Potência (Equação 1):

'(Trabalho 143-07).Recebido em: 06-06-2007. Aceito para publicação em: 22-02-2008.

${ }^{2}$ Doutorando do Programa de Pós-graduação em Tecnologia de Processos Químicos e Bioquímicos, Escola de Química, UFRJ, Bolsista CNPq, e-mail: mmgilv@yahoo.com.

${ }^{3}$ Professor adjunto, Departamento de Processos Orgânicos da Escola de Química, UFRJ. e-mail: mjg@eq.ufrj.br.

${ }^{4}$ Professor adjunto, Departamento de Engenharia Bioquímica da Escola de Química, UFRJ. e-mail: antun@eq.ufrj.br. 


$$
\tau=\mathrm{K} \gamma^{\mathrm{n}}
$$

O modelo de Herschel-Bulkley (Equação 2) é derivado do modelo Ostwald-de-Waelle adicionado do termo de tensão inicial $\left(\tau_{\mathrm{o}}\right)$ :

$$
\tau=\tau_{\mathrm{o}}+\mathrm{K} \gamma^{\mathrm{n}}
$$

onde: $\mathrm{K}=$ índice de consistência $\left(\mathrm{Pa} \cdot \mathrm{s}^{\mathrm{n}}\right) ; \tau_{\mathrm{o}}=$ tensão de cisalhamento inicial $(\mathrm{Pa}) ; \tau=$ tensão de cisalhamento $(\mathrm{Pa}) ; \gamma=$ taxa de deformação $\left(\mathrm{s}^{-1}\right) ; \mathrm{n}=$ índice de comportamento de fluxo (adimensional).

Os modelos de Ostwald-de-Waelle e Herschel-Bulkley são muito usados, e dados reológicos para fluidos alimentícios não-newtonianos têm sido publicados na literatura (Akdogan \& Mchugh, 2000; Dak et al., 2006; Gratão et al., 2007).

Em geral, o efeito da temperatura na viscosidade aparente $\left(\eta_{\mathrm{a}}\right)$, a uma taxa de deformação específica, tem sido expresso por uma equação do tipo Arrhenius (Altan \& Maskan, 2005; Grigelmo-Miguel et al., 1999; Magerramov et al., 2007; Togrul \& Arslan, 2004), como mostrada na Equação 3.

$$
\eta_{\mathrm{a}}=\operatorname{Aexp}\left(\mathrm{E}_{\mathrm{a}} / \mathrm{RT}\right)
$$

onde: $\mathrm{A}=$ constante empírica (Pa.s); $\mathrm{E}_{\mathrm{a}}=$ energia de ativação do fluxo (kcal/gmol);

$\mathrm{R}=$ constante do gás ideal $\left(1,987 \times 10^{-3} \mathrm{kcal} / \mathrm{gmol} . \mathrm{K}\right) ; \mathrm{T}=$ temperatura absoluta $(\mathrm{K})$.

A equação de Arrhenius indica a dependência da viscosidade aparente com a temperatura. A ordem de grandeza da energia de ativação mostra a dependência da viscosidade com a temperatura, sendo que o aumento da temperatura provoca um efeito de decréscimo da viscosidade (Silva, 2000).

Trabalhos relativos às frutas tropicais originárias do Norte brasileiro, principalmente o cupuaçu, inclusive com respeito à determinação de suas propriedades físicas e, em particular, ao comportamento reológico de seus derivados, são ainda escassos. Assim, o objetivo deste trabalho foi realizar um estudo do comportamento reológico em estado estacionário da polpa de cupuaçu integral na faixa de temperatura de 10 a $60^{\circ} \mathrm{C}$, analisando também o efeito da taxa de deformação empregada.

\section{MATERIAL E MÉTODOS}

A polpa de cupuaçu a $9^{\circ}$ Brix e $\mathrm{pH} 3,3$ foi obtida da Cooperativa dos Produtores de Cupuaçu do Pará. Foi acondicionada em sacos de polietileno, estocada e congelada à temperatura de $-20^{\circ} \mathrm{C}$ em freezer ,até o momento da análise.

No estudo reológico em cisalhamento estacionário, utilizou-se o reômetro Thermo Haake modelo RheoStress 1, equipado com célula de Couette RS1 Z20 Din (30 mm de altura; $20 \mathrm{~mm}$ e 21,7 mm de diâmetro interno e externo, respectivamente). O tempo de cada corrida foi de 4 minutos, em taxa crescente $(0,3$ a $\left.300 \mathrm{~s}^{-1}\right)$ e taxa decrescente $\left(300\right.$ a $\left.0,3 \mathrm{~s}^{-1}\right)$.

As medidas foram realizadas nas temperaturas de $10 ; 20$; $30 ; 40 ; 50$ e $60^{\circ} \mathrm{C}$ por serem, a primeira e a última, a temperatura típica de prateleira do produto acabado e a temperatura representativa de pasteurização nas indústrias.

Os dados de tensão e a taxa de deformação obtidos foram ajustados aos modelos de escoamento de Herschel-Bulkley e Ostwald-de-Waelle através do software Origin 5.0, tendo como parâmetros estatísticos os valores de $\mathrm{R}^{2} \mathrm{e} \chi^{2}$.

- Coeficiente de determinação $\left(\mathrm{R}^{2}\right)$ - Mede a proporção da variação total da média explicada pela regressão, definida como a soma quadrática total.

- Qui-quadrado $\left(\chi^{2}\right)$ - Expressa a diferença entre os valores previstos pelo modelo e os valores obtidos experimentalmente.

\section{RESULTADOS E DISCUSSÃO}

Os parâmetros de escoamento relativos aos modelos de Herschel-Bulkley e Ostwald-de-Waelle estão mostrados na Tabela 1. A Figura 1 apresenta as curvas de escoamento, e a Figura 2 apresenta as curvas de viscosidade.

O modelo de Herschel-Bulkley (HB) ajustou-se bem aos dados experimentais, no entanto observa-se que, na temperatura de $60^{\circ} \mathrm{C}$, o valor de tensão residual foi negativo e, portanto, sem significado físico.

O modelo de Ostwald-de-Waelle (LP) adequou-se de modo satisfatório em todas as temperaturas analisadas, apresentando baixos valores de $\chi^{2}$ e altos valores de $\mathrm{R}^{2}$.

$\mathrm{O}$ índice de comportamento de fluxo (n) foi inferior a 1 para todas as amostras, caracterizando, dessa forma, um comportamento não-newtoniano, corroborando os resultados de Ahmed et al. (2005) e Conceição (2000), ao estudarem o comportamento reológico da polpa de manga e da polpa de goiaba integral. Foi observado um decréscimo de $54,76 \%$ no índice de comportamento entre as temperaturas de $10^{\circ} \mathrm{C}$ e $60^{\circ} \mathrm{C}$.

Nas Figuras 1 e 2, é apresentada a influência da temperatura no comportamento reológico da polpa de cupuaçu. Nelas, são representados os pontos experimentais médios e suas respectivas curvas de ajuste ao modelo de Ostwald-de-Waelle. Observa-se que, para uma taxa de deformação fixa, a tensão de cisalhamento diminui à medida que aumenta a temperatura (Figura 1). Comportamento semelhante foi encontrado por Nindo et al. (2007), Harnanan et al. (2001) e Ferreira et al. (2003), ao estudarem as propriedades reológicas do purê de mirtilo e polpa de goiaba. Pode-se observar também que a viscosidade aparente (Figura 2) decresce com o aumento da taxa de deformação, indicando um comportamento pseudoplástico para a polpa. Esse tipo de comportamento tem sido encontrado por diversos autores, entre os quais, Arslan et al. (2005), Kaya \& Belibagli (2002) e Pelegrine et al. (2002), ao analisarem suco de uva concentrado e as polpas de manga e abacaxi, respectivamente.

$\mathrm{O}$ decréscimo da viscosidade aparente com o aumento da taxa de deformação pode ser explicado pela mudança estrutural da amostra devido às forças hidrodinâmicas geradas e o maior alinhamento das moléculas na direção da tensão aplicada (Alparslan \& Hayta, 2002).

Mediante um ajuste não-linear dos dados de viscosidade aparente $\left(\eta_{\mathrm{a}}\right)$ da Tabela 2 e utilizando a equação de Arrhenius (Eq. 3), que relaciona o efeito da temperatura na viscosidade de 
diversos alimentos líquidos, determinaram-se os valores de energia de ativação e do parâmetro $\eta_{0}$ da polpa, nas temperaturas de $10^{\circ} \mathrm{C}$ a $60^{\circ} \mathrm{C}$. Os parâmetros estão apresentados na Tabela 3.

Na Tabela 2 e Figura 3, são apresentados os valores de viscosidade aparente da polpa de cupuaçu para taxas de deformação de $100 ; 200$ e $300 \mathrm{~s}^{-1}$, cujos valores são importantes nas operações industriais de processamento, onde a taxa de deformação é sempre maior que $100 \mathrm{~s}^{-1}$ (Bezerra, 2000). Como já observado na Figura 2, verifica-se o decréscimo da viscosidade aparente, considerando isoladamente os aumentos de temperatura e da taxa de deformação.

Sengul et al. (2005) observaram comportamento semelhante para sucos de uva e amora concentrados. Lopes (2005), analisando o comportamento reológico da polpa de pitanga, observou um decréscimo da viscosidade aparente com o aumento da temperatura e da taxa de deformação.

A equação de Arrhenius apresentou um bom ajuste do efeito da temperatura na viscosidade aparente da polpa nas taxas de deformação utilizadas, como pode ser visto na Figura 3 e
Tabela 3. O valor da energia de ativação (Ea) aumentou com o aumento da taxa de cisalhamento e encontra-se na faixa de valores encontrados por Alexandre (2002), ao analisar o comportamento reológico da polpa de açaí integral, cujo valor foi igual a $1,5 \mathrm{kcal} /$ gmol, ao de Ferreira (2002), ao analisar o comportamento reológico da polpa de goiaba integral $(\mathrm{Ea}=1,47 \mathrm{kcal} / \mathrm{gmol})$ e por Guerrero \& Alzamora (1997) $(\mathrm{Ea}=1,91 \mathrm{kcal} / \mathrm{gmol})$, ao estudar o comportamento do purê de banana. Essa faixa de valores para energia de ativação é característica dos sistemas com altos teores de sólidos em suspensão.

Como esperado, para polpas de frutas, nota-se que a viscosidade aparente diminuiu com o aumento da taxa de deformação devido ao maior alinhamento das partículas na direção da tensão aplicada, enquanto a energia de ativação (Ea) aumentou com o aumento da taxa de deformação. Maiores valores de energia de ativação (Ea) indicam uma mudança mais rápida na viscosidade com a temperatura, como mostra a Figura 3.

TABELA 1 - Parâmetros reológicos para a polpa de cupuaçu.

\begin{tabular}{|c|c|c|c|c|c|c|c|c|c|c|}
\hline \multirow[b]{2}{*}{ Amostra } & \multirow[b]{2}{*}{$\begin{array}{l}\mathrm{T} \\
\left({ }^{\circ} \mathrm{C}\right)\end{array}$} & \multicolumn{2}{|c|}{ Ostwald-de-Waelle } & \multirow[b]{2}{*}{$\overline{\mathbf{R}^{2}}$} & \multirow[b]{2}{*}{$\chi^{2}$} & \multicolumn{3}{|c|}{ Herschel-Bulkley } & \multirow[b]{2}{*}{$\mathbf{R}^{2}$} & \multirow[b]{2}{*}{$\chi^{2}$} \\
\hline & & $\begin{array}{l}\text { K } \\
(\text { Pa.s })^{n}\end{array}$ & n & & & $\begin{array}{l}\tau_{0} \\
(\mathbf{P a})\end{array}$ & $\begin{array}{l}\text { K } \\
(\text { Pa.s })^{n}\end{array}$ & n & & \\
\hline \multirow[t]{6}{*}{ Cup } & 10 & 3,09 & 0,42 & 0,99 & 0,268 & 6,58 & 0,89 & 0,60 & 0,99 & 0,054 \\
\hline & 20 & 2,73 & 0,42 & 0,98 & 0,656 & 8,66 & 0,28 & 0,77 & 0,99 & 0,107 \\
\hline & 30 & 4,84 & 0,31 & 0,98 & 0,474 & 9,58 & 0,62 & 0,60 & 0,98 & 0,245 \\
\hline & 40 & 3,88 & 0,33 & 0,94 & 1,149 & 10,31 & 0,14 & 0,82 & 0,96 & 0,741 \\
\hline & 50 & 4,51 & 0,29 & 0,97 & 0,381 & 2,31 & 3,15 & 0,34 & 0,97 & 0,385 \\
\hline & 60 & 5,37 & 0,23 & 0,96 & 0,288 & $-5,10$ & 9,32 & 0,17 & 0,96 & 0,290 \\
\hline
\end{tabular}

TABELA 2 - Viscosidade aparente da polpa de cupuaçu em diferentes taxas de deformação.

\begin{tabular}{llll}
\hline & Viscosidade aparente (mPa.s) & \\
Temperatura $\left({ }^{\mathbf{O}} \mathbf{C}\right)$ & $\mathbf{1 0 0} \mathbf{s}^{\mathbf{1}}$ & $\mathbf{2 0 0} \mathbf{s}^{-\mathbf{1}}$ & $\mathbf{3 0 0 ~ s}^{\mathbf{- 1}}$ \\
\hline 10 & 213,77 & 143,01 & 113,04 \\
20 & 188,87 & 126,35 & 99,87 \\
30 & 201,76 & 125,06 & 94,54 \\
40 & 177,35 & 111,47 & 84,95 \\
50 & 171,46 & 104,82 & 78,59 \\
60 & 154,87 & 90,82 & 66,46 \\
\hline
\end{tabular}

TABELA 3 - Parâmetros da Equação de Arrhenius para a polpa de cupuaçu em diferentes taxas de deformação.

\begin{tabular}{llcc}
\hline & Taxa de deformação $\left.\mathbf{( s}^{-\mathbf{1}}\right)$ & \\
$\begin{array}{l}\text { Parâmetros } \\
\text { de Arrhenius }\end{array}$ & $\mathbf{1 0 0}$ & $\mathbf{2 0 0}$ & $\mathbf{3 0 0}$ \\
\hline $\mathrm{A}(\mathrm{mPas})$ & & 9,57 & 4,68 \\
$\mathrm{Ea}(\mathrm{kcal} / \mathrm{gmol})$ & 32,78 & 1,52 & 1,79 \\
$\mathrm{R}^{2}$ & 1,05 & 0,96 & 0,98 \\
\hline
\end{tabular}




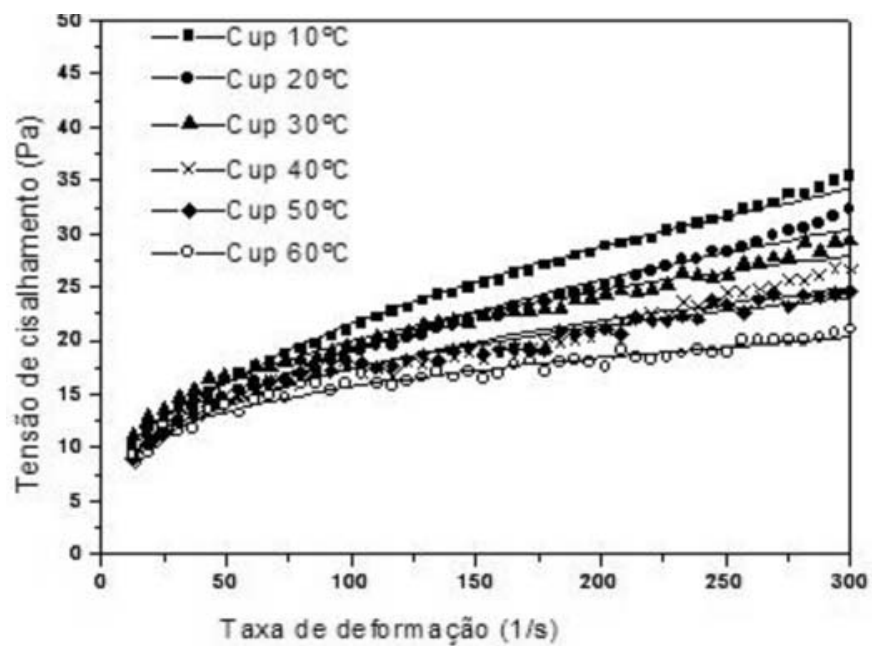

FIGURA 1 - Propriedades reológicas em função da temperatura - curvas de escoamento.

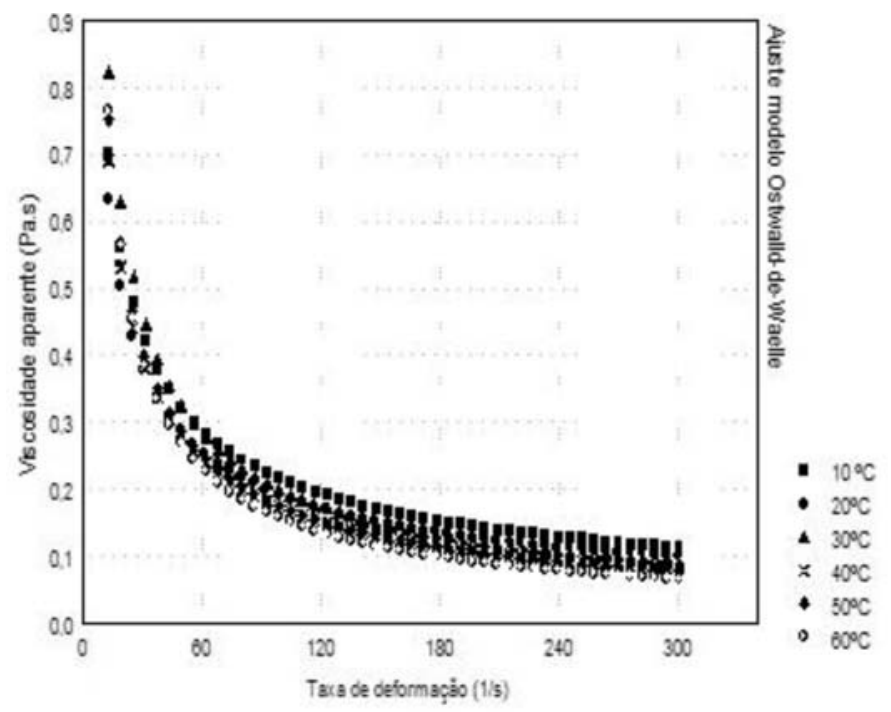

FIGURA 2 - Propriedades reológicas em função da temperatura - curvas de viscosidade.

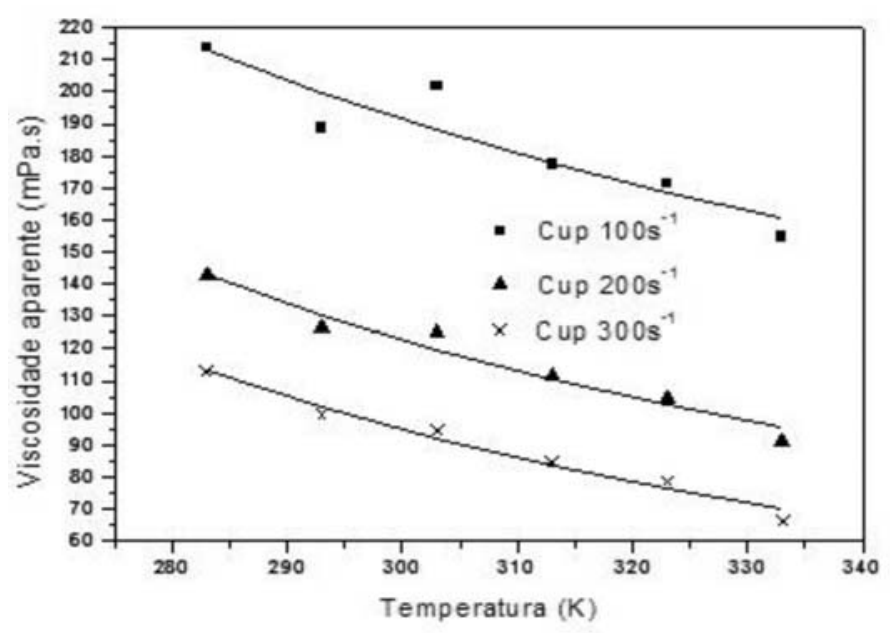

FIGURA 3 - Influência da temperatura na viscosidade aparente para a polpa de cupuaçu.

\section{CONCLUSÕES}

1- Foram testados os modelos de Herschel-Bulkley e de Ostwald-de-Waelle, sendo o modelo Ostwald-de-Waelle o que melhor se ajustou aos dados experimentais.

2- O índice de comportamento de fluxo (n) foi inferior a 1 , indicando um comportamento não-newtoniano com características pseudoplásticas.

3- A dependência da viscosidade aparente em função da temperatura, foi bem representada por uma Equação de Arrenhius.

4- A energia de ativação para a polpa aumentou com o aumento da taxa de cisalhamento e foram obtidos valores na faixa de 1 a $2 \mathrm{kcal} / \mathrm{gmol}$.

\section{AGRADECIMENTOS}

Os autores agradecem ao CNPq, pelo apoio financeiro, e à Dra. Christine Rabello Nascimento, do CETEM, pelo treinamento do reômetro.

\section{REFERÊNCIAS}

AHMED, J.; RAMASWAMY, H.S.; HIREMATH, N. The effect of high pressure treatment on rheological characteristics and colour of mango pulp. International Journal of Food Science and Technology, Oxford, v.40, p.885-895, 2005.

ALEXANDRE, D. Conservação da polpa de açaí através da tecnologia de obstáculos e caracterização reológica. 2002. 161f. Dissertação (Mestrado em Engenharia de Alimentos) Universidade Estadual de Campinas, Campinas. 2002.

AKDOGAN, H.; MCHUGH, T.H. Flow characterization of peach products during extrusion. Journal of Food Science, Chicago, v. 65, n. 3, p. $471-475,2000$.

ALTAN, A.; MASKAN, M. Rheological behavior of pomegranate (Punica granatum L.) juice and concentrate. Journal of Texture Studies, Trumbull, v.36, p.68-77, 2005.

ALPARSLAN, M.; HAYTA, M. Rheological and sensory properties of pekmez (grape molasses)/tahin(sesame paste) blends. Journal of Food Engineering, Oxford, v.54, n.1, p.89-93, 2002.

ARSLAN, E.; YENER, M.E.; ESIN, A. Rheological characterization of tahin/pekmez (sesame paste/concentrated grape juice) blends. Journal of Food Engineering, Oxford, v.69, p. 167-172, 2005.

BASTOS, M.S.R.; GURGEL, T.E.P.; SOUSAFILHO, M.S.M. Efeito da aplicação de enzimas pectinolíticas no rendimento da extração de polpa de cupuaçu. Revista Brasileira de Fruticultura, Jaboticabal, v.24, n.1, p.240-242, 2002. 
BEZERRA, J.R.M.V. Comportamento da polpa de manga. 2000. 159f. Tese (Doutorado em Engenharia de Alimentos) Universidade Estadual de Campinas, Campinas, 2000.

CONCEIÇÃO, R.S. Comportamento reológico da polpa de goiaba (Psidium guajava L.). 2000. 68f. Dissertação (Mestrado em Engenharia Agrícola) - Universidade Federal da Paraíba, Paraíba, 2000.

DAK, M.; VERMA, R.C.; SHARMA, G.P. Flow characteristics of juice of "Totapuri" mangoes. Journal of Food Engineering, Oxford, v.76, n.4, p.557-561, 2006.

FERREIRA, G.M.; QUEIROZ, A.J. de M.; CONCEIÇÃO, R.S.; GASPARETTO, C.A., Reologia de misturas de polpas de goiaba e caju peneiradas. Revista Estudos Tecnológicos - Engenharia, São Leopoldo, v.14, n.25, p.49-55, 2003.

FERREIRA, G.M. Reologia de polpas de caju (Anacardium occidentale L.) e goiaba (Psidium guajava L.). 2002. $101 \mathrm{f}$. Dissertação (Mestrado em Engenharia Agrícola) - Universidade Federal da Paraíba, Paraíba, 2002.

FRANCO, G. Tabela de composição química dos alimentos. 9. ed. São Paulo: Ateneu, 1999. 307p.

GRATÃO, A.C.A.; SILVEIRA Jr, V.; TELIS-ROMERO, J. Laminar flow of soursop juice through concentric annuli: friction factors and rheology. Journal of Food Engineering, Oxford, v.78, p.13431354, 2007.

GRIGELMO-MIGUEL, N.; IBARZ-RIBAS, A.; MARTINBELLOSO, O. Rheology of peach dietary fibre suspensions. Journal of Food Engineering, Oxford, v.39, n.1, p.91-99, 1999.

GUERRERO, S.N.; ALZAMORA, S.M. Effect of $\mathrm{pH}$, temperature and glucose addition on flow behavior of fruit purées I. Banana Purée. Journal of Food Engineering, Oxford, v.33, p.239-256, 1997.

HARNANAN, S.W.; TEJINDER, S.; BAINS, G.S. Effect of processing, preservation and storage on rheology of guava pulp. Journal of Texture Studies, Trumbull, v.32, p.271-284, 2001.

KAYA, A.; BELIBAGLI, K.B. Rheology of solid Gaziantep Pekmez. Journal of Food Engineering, Oxford, v.54, p.221-226, 2002.

LOPES, A.S. Pitanga e acerola: estudo de processamento, estabilidade e formulação de néctar misto. 2005. 137f. Tese
(Doutorado em Engenharia de alimentos) - Universidade Estadual de Campinas, Campinas. 2005.

LOPES, A.S. Estudo químico e nutricional de amêndoas de cacau (Theobroma cacao L.) e cupuaçu (Theobroma grandiflorum Schum) em função do processamento. 2000. 112f. Dissertação (Mestrado em Engenharia de Alimentos) - Universidade Estadual de Campinas, Campinas, 2000.

MAGERRAMOV, M.A.; ABDULAGATOV, A.I.; AZIZOV, N.D.; ABDULAGATOV, I.M. Effect of temperature, concentration and pressure on the viscosity of pomegranate and pear juice concentrates. Journal of Food Engineering, Oxford, v.80, p.476489, 2007.

NINDO, C.I.; TANG J.; POWERS, J.R.; TAKHAR, P.S. Rheological properties of blueberry puree for processing applications. Lebensmittel Wissenschaft und-Technologie, Oxford, v.40, p.292-299, 2007.

PELEGRINE, D.H.; SILVA, F.C.; GASPARETTO, C.A. Rheological behavior of pineapple and mango pulps. Lebensmittel Wissenschaft und-Technologie, Oxford, v.35, n.8, p.645-648, 2002.

SAGRI. Secretaria executiva de agricultura do Pará (2006). Disponível em: <www.sagri.pa.gov.br/dados.htm>. Acesso em: 20 out. 2007.

SENGUL, M.; ERTUGAY, M.F.; SENGUL, M. Rheological, physical and chemical characteristics of mulberry pekmez. Food Control, Guildford, v.16,p.73-76, 2005.

SILVA, F.C. Reologia do suco de acerola: efeito da concentração e da temperatura. 2000. 110f. Dissertação (Mestrado em Engenharia de Alimentos) - Universidade Estadual de Campinas, Campinas, 2000.

STEFFE, J.F. Rheological methods in food process engineering $2^{\text {nd }}$ ed. Michigan: Freeman Press, 1996. 428p.

TOGRUL, H.; ARSLAN, N. Mathematical model for prediction of apparent viscosity of molasses. Journal of Food Engineering, Oxford, v.62, p.281-289, 2004.

VENTURIERI, G.A. Cupuaçu: a espécie, sua cultura, usos e processamento. Belém: Clube do Cupu, 1993. 108p. 